\title{
A Nomogram for Predicting In-Stent Restenosis Risk in Patients Undergoing Percutaneous Coronary Intervention: A Population-Based Analysis
}

\author{
Yinhua Luo',*, Ni Tan ${ }^{2} *$, Jingbo Zhao ${ }^{3}$, Yuanhong $\mathrm{Li}^{3}$ \\ 'Department of Central Hospital of Tujia and Miao Autonomous Prefecture, Hubei University of Medicine, Shiyan, Hubei Province, 442000, People's \\ Republic of China; ${ }^{2}$ Pulmonary and Critical Care Medicine, Central Hospital of Tujia and Miao Autonomous Prefecture, Hubei University of Medicine, \\ Enshi Prefecture, Hubei Province, 445000, People's Republic of China; ${ }^{3}$ Cardiovascular Disease Center, Central Hospital of Tujia and Miao \\ Autonomous Prefecture, Enshi Prefecture, Hubei Province, 445000, People's Republic of China
}

*These authors contributed equally to this work

Correspondence: Yuanhong Li, Cardiovascular Disease Center, Central Hospital of Tujia and Miao Autonomous Prefecture, Hubei University of Medicine, Enshi Prefecture, People's Republic of China, Email lyh0I0I@vip.I63.com

Objective: In-stent restenosis (ISR) is a fatal complication of percutaneous coronary intervention (PCI). An early predictive model with the medical history of patients, angiographic characteristics, inflammatory indicators and blood biochemical index is urgently needed to predict ISR events. We aim to establish a risk prediction model for ISR in CAD patients undergoing PCI.

Methods: A total of 477 CAD patients who underwent PCI with DES (drug-eluting stents) between January 2017 and December 2020 were retrospectively enrolled. And the preoperative factors were compared between the non-ISR and ISR groups. The least absolute shrinkage and selection operator (LASSO) and multi-factor logistic regression were used for statistical analysis. The prediction model was evaluated using receiver operator characteristic (ROC) analysis, the Hosmer-Lemeshow 2 statistic, and the calibration curve.

Results: In this study, 94 patients developed ISR after PCI. Univariate analysis showed that post-PCI ISR was associated with the underlying disease (COPD), higher Gensini score (GS score), higher LDL-C, higher neutrophil/lymphocyte ratio, and higher remnant cholesterol (RC). The multi-factor logistic regression analysis suggested that remnant cholesterol (odds ratio [OR] $=2.09$, 95\% confidence interval [CI] [1.40-3.11], P $<0.001)$, GS score $(\mathrm{OR}=1.01,95 \% \mathrm{CI}[1.00,1.02], \mathrm{P}=0.002)$, medical history of COPD $(\mathrm{OR}=4.56,95 \% \mathrm{CI}[1.98,10.40], \mathrm{P}<0.001)$, and monocyte $(\mathrm{OR}=1.30,95 \% \mathrm{CI}[1.04,1.70], \mathrm{P}<0.001)$ were independent risk factors for ISR. A nomogram was generated and displayed favorable fitting (Hosmer-Lemeshow test $\mathrm{P}=0.609$ ), discrimination (area under ROC curve was 0.847 ), and clinical usefulness by decision curve analysis.

Conclusion: Patients with certain preoperative characteristics, such as a history of COPD, higher GS scores, higher levels of RC, and monocytes, who undergo PCI may have a higher risk of developing ISR. The predictive nomogram, based on the above predictors, can be used to help identify patients who are at a higher risk of ISR early on, with a view to provide post-PCI health management for patients.

Keywords: in-stent restenosis, ISR, percutaneous coronary intervention, PCI, coronary heart disease, CHD, nomogram map

\section{Introduction}

Coronary heart disease (CHD), with high morbidity and high mortality rate, is still a serious public health concern around the world. PCI is fast becoming a key instrument in revascularization for patients with $\mathrm{CHD}$, as well as an important technology in the management of CHD patients. ${ }^{1}$ Although the clinical application of coronary stents brought about a dramatic improvement in patients' clinical and procedural outcomes, the mid-and long-term outcome of stent implantation remains significantly hampered by the risk of developing ISR with a prevalence rate of $3-20 \%$ over time ${ }^{1,2}$ Predictive models have the advantage of formally combining risk factors to allow more accurate risk estimation. And it is essential to establish a model to predict ISR in patients with CAD and drug-eluting stents (DESs) implantation. 
The risk factors for ISR after PCI were systematically summarized. The preoperative factors comprised the following: the morphological characteristics of the diseased vessel, the location of the lesion, the degree of stenosis, and part of blood biochemical indicators which are associated with inflammatory responses and lipid metabolism. ${ }^{3-8}$ Among these, the levels of monocyte and LDL-C are considered to be critical factors related to inflammatory response and to lipid metabolism, ${ }^{9-11}$ respectively.

Although a few previous studies have analyzed potential predictors related to the high incidence rate of ISR and established a relevant nomogram for ISR in patients undergoing PCI, there are still limitations to the predictive model. As a starting point, new factors for inflammatory response and lipid metabolism have emerged in recent years. Such as the neutrophil/lymphocyte ratio ${ }^{12,13}$, which reflects the body's levels of oxidative stress and inflammation, as well as residual cholesterol, which is a more accurate indicator of the body's lipid metabolism than LDL cholesterol. ${ }^{14,15}$ Secondly, the majority of prediction models lacked a quantitative predictor of coronary lesions before PCI, such as the GS score system, a technique based on the artery morphology, coronary anatomy, and severity of stenosis in lesions.

Currently, a new preoperative model based on preoperative blood biochemical parameters for PCI, a technique for assessment for the severity of CAD, and procedural characteristics are scarce to evaluate the probability of ISR. The aim of this study was to analyzed post-PCI ISR patients in preoperative blood biochemical parameters for PCI, GS scores, ${ }^{16}$ and procedural characteristics. This work will generate fresh insight into developing a preoperative risk factor nomogram that may help clinicians discern high-risk ISR patients, optimize treatment strategies.

\section{Materials and Methods}

According to the Declaration of Helsinki, this study was approved by the Ethics Committee of the Central Hospital of Enshi Autonomous Prefecture. Due to the retrospective nature of this study, patient consent was waived for the evaluation of their medical information. This study was an analysis of an observational cohort study conducted from January 2017 to December 2020 at Enshi Central Hospital, China. A total of 1015 CAD patients undergoing PCI with DES were enrolled. All patients took statins and anti-platelet aggregation drugs regularly after surgery, and all received 6-24 months of follow-up coronary angiography. Patients were excluded if they (1) had a history of coronary artery bypass grafting, heart failure (cardiac function class more than 4), (2) active or acute inflammatory diseases, (3) had a liver failure or renal failure, (4) had evidence of active infection, such as fever, cough, or diarrhea, and (5) were missing clinical and angiographic data (Figure 1). The main outcome measure was ISR, which is defined as $\geq 50 \%$ luminal narrowing at follow-up angiography.

Demographic information, biochemical parameters, clinical, and angiographic characteristics were collected. Demographic information and clinical characteristics included age, gender, chronic obstructive pulmonary disease, diabetes, stroke, smoking, and patient medication history (ACEI, diuretic). Biochemical parameters included platelet parameters of platelet distribution width (PDW), leukocytes, monocytes, mean platelet volume (MPV), glucose (GLU), neutrophils $(\mathrm{N})$, lymphocyte, monocyte, hemoglobin $(\mathrm{Hb})$, platelet, procalcitonin (PCT), total cholesterol (TC), triglycerides (TG), high-density lipoprotein cholesterol (HDL-C), low-density lipoprotein cholesterol (LDL-C), the width of red blood cell volume distribution (RDW), alanine transaminase (ALT), creatinine (Cr) and left ventricular systolic function $(\mathrm{EF} \%)$. The specific data concerning the angiography information included stent numbers, GS score, lesion location (left main coronary artery, left circumflex artery, left anterior descending branch, right coronary artery and others. RC = TC- LDL-C- HDL-C. The neutrophil/lymphocyte ratio = the neutrophil count /the lymphocyte count. The GS score was calculated using the scoring schema defined by Gensini et al. ${ }^{16}$

$\mathrm{R}$ software version 3.6.3 was used for statistical analysis, and two-tailed analysis with P. The Student's $t$-test was used to detect differences between continuous variables with a normal distribution. The chi-square test or Fisher's exact test was used to compare categorical variables. In the cases of skewed distribution, data were expressed as IQR and compared using the Mann-Whitney $U$-test. LASSO-penalized regression analysis, which is capable of estimating parameters in high-dimensional regression, was used to select ISR predictors with the R package Glmnet. The Hosmer-Lemeshow 2 statistic, calibration curve, and 1000-fold bootstrap were used to test the prediction model.

We assessed the nomogram model performance in terms of discrimination, calibration plots, and the HosmerLemeshow 2 statistic. The discrimination of the model has been validated through Area under the ROC, which implies 


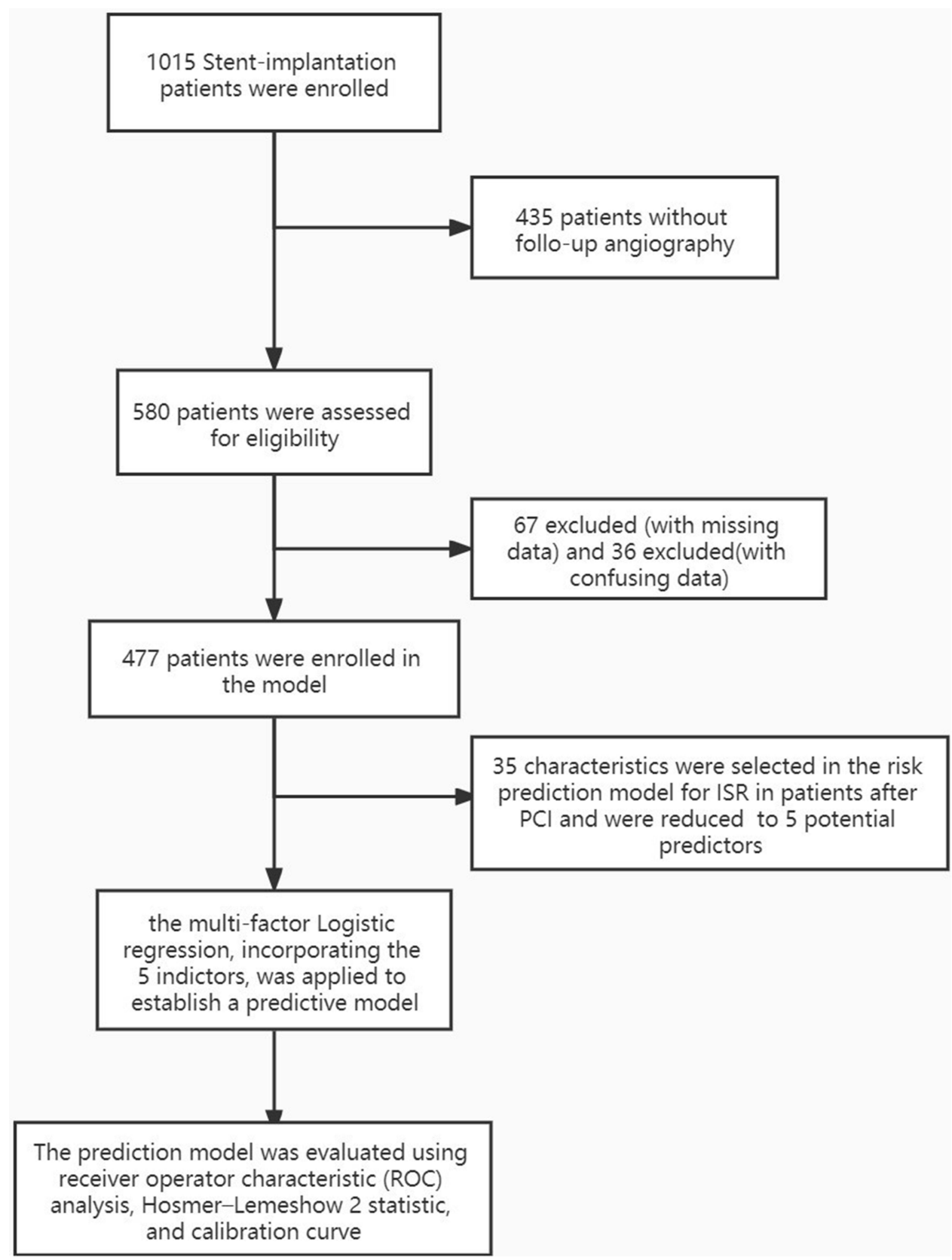

Figure I The study design and the selection procession of CAD patients.

Abbreviation: receiver operator characteristic.

the better accuracy of the nomogram. The diagnostic value of models whose AUC is between 0.7 and 0.8 and HosmerLemeshow $2>0.05$ is acceptable A very perfect agreement was observed in the calibration plot of our nomogram. Concerning its clinical usefulness, we performed decision curve analysis (DCA) to assess if clinical decisions taken based on this model would improve patient outcomes.

\section{Results}

According to the inclusion and exclusion criteria, 1015 patients were screened, and 477 patients with complete follow-up data were selected. A total of 477 patients was enrolled and divided into the ISR group (94) and the non-ISR group (383), according to the main outcome indicator (ISR). The baseline characteristics of the patients are shown in Table 1. 
Table I The Baseline Characteristics of the Patients

\begin{tabular}{|c|c|c|c|}
\hline Characteristics & ISR=No $(n=383)$ & ISR=Yes $(n=94)$ & P-value \\
\hline Gender, N (\%) & & & 0.977 \\
\hline Female & $82(21.4)$ & $20(21.3)$ & \\
\hline Male & $301(78.6)$ & $74(78.7)$ & \\
\hline Hypertension, N (\%) & & & 0.961 \\
\hline No & $194(50.8)$ & $48(5 I .1)$ & \\
\hline Yes & $188(49.2)$ & $46(48.9)$ & \\
\hline COPD, N (\%) & & & $<0.001$ \\
\hline No & $368(96.1)$ & $80(85.1)$ & \\
\hline Yes & $15(3.9)$ & $14(14.9)$ & \\
\hline Diabete, N (\%) & & & 0.342 \\
\hline No & $325(84.9)$ & $76(80.9)$ & \\
\hline Yes & $58(15.1)$ & $18(19.1)$ & \\
\hline Stroke, N (\%) & & & 0.389 \\
\hline No & $377(98.4)$ & $91(96.8)$ & \\
\hline Yes & $6(1.6)$ & $3(3.2)$ & \\
\hline Smoking, N (\%) & & & 0.226 \\
\hline No & $169(44.1)$ & $35(37.2)$ & \\
\hline Yes & $214(55.9)$ & $59(62.8)$ & \\
\hline Multi_vessel, N (\%) & & & 0.84 \\
\hline No & $102(26.6)$ & $26(27.7)$ & \\
\hline Yes & $281(73.4)$ & $68(72.3)$ & \\
\hline Left main coronary artery, $\mathrm{N}(\%)$ & & & 0.259 \\
\hline No & $344(89.8)$ & $88(93.6)$ & \\
\hline Yes & $39(10.2)$ & $6(6.4)$ & \\
\hline Left circumflex artery, $\mathrm{N}(\%)$ & & & 0.264 \\
\hline No & $163(42.6)$ & $46(48.9)$ & \\
\hline Yes & $220(57.4)$ & $48(51.1)$ & \\
\hline Left anterior descending branch, $\mathrm{N}(\%)$ & & & 0.631 \\
\hline No & $46(12)$ & $13(13.8)$ & \\
\hline Yes & $337(88)$ & $81(86.2)$ & \\
\hline Right coronary artery and others, $\mathrm{N}(\%)$ & & & 0.401 \\
\hline No & $137(35.8)$ & $38(40.4)$ & \\
\hline Yes & $246(64.2)$ & $56(59.6)$ & \\
\hline ACEI, N (\%) & & & 0.113 \\
\hline No & $70(18.3)$ & $24(25.5)$ & \\
\hline Yes & $313(8 \mid .7)$ & $70(74.5)$ & \\
\hline Diuretic, N (\%) & & & 0.352 \\
\hline No & $321(83.8)$ & $75(79.8)$ & \\
\hline Yes & $62(16.2)$ & $19(20.2)$ & \\
\hline Age, median(IQR) & $61(54,68)$ & $63(55,68)$ & 0.48 \\
\hline The_number_of_stents, median(IQR) & I $(I, 2)$ & I $(I, 2)$ & 0.772 \\
\hline GS_grade, median(IQR) & $40(22.5,68)$ & $48(38,80.8)$ & $<0.001$ \\
\hline Leukocyte(I0^9/L), median(IQR) & $7.2(5.8,8.9)$ & $6.8(5.6,8.3)$ & 0.172 \\
\hline$N\left(I 0^{\wedge} 9 / L\right), \operatorname{median}(I Q R)$ & $4.6(3.5,6.4)$ & $4.6(3.6,6.3)$ & 0.623 \\
\hline Lymphocyte(I0^9/L), median(IQR) & $1.6(1.2,2)$ & $1.6(1,2.1)$ & 0.902 \\
\hline Monocyte(I0^9/L), median(IQR) & $0.4(0.3,0.5)$ & $0.4(0.4,0.6)$ & 0.112 \\
\hline $\mathrm{Hb}(\mathrm{g} / \mathrm{L})$, median $(\mathrm{IQR})$ & $136(124,147)$ & | $38.5(|29.2| 48.8)$ & 0.474 \\
\hline PDW & $16.4(16.1,16.6)$ & $16.4(16.2,16.6)$ & 0.415 \\
\hline Platelet(I0^9/L), median(IQR) & $188(\mid 57,227.5)$ & $183.5(162.2213)$ & 0.473 \\
\hline MPV(fl), median(IQR) & $10.8(9.9,11.7)$ & $10.4(9.6,11.7)$ & 0.256 \\
\hline $\mathrm{PCT}$, median(IQR) & $0.2(0.2,0.2)$ & $0.2(0.2,0.2)$ & 0.716 \\
\hline
\end{tabular}

(Continued) 
Table I (Continued).

\begin{tabular}{|c|c|c|c|}
\hline Characteristics & ISR=No $(n=383)$ & ISR=Yes $(n=94)$ & P-value \\
\hline RDW, median(IQR) & $12.9(12.5,13.3)$ & $12.8(12.4,13.3)$ & 0.295 \\
\hline ALT, median(IQR) & $27(18,43)$ & $27(21,39)$ & 0.963 \\
\hline TC, median(IQR) & $4.7(4.1,5.4)$ & & 0.062 \\
\hline $\mathrm{TG}, \operatorname{median}(\mathrm{IQR})$ & $1.6(1.1,2.2)$ & $\mathrm{I} .5(\mathrm{I} . \mathrm{I}, 2)$ & 0.506 \\
\hline $\mathrm{HDL} C \mathrm{C}(\mathrm{mmol} / \mathrm{L}), \mathrm{IQR}]$ & I $(0.9, \mid .2)$ & I.I $(0.8,1.2)$ & 0.917 \\
\hline LDL_C[(mmol/L), IQR] & $3(2.5,3.5)$ & $2.6(2,3.1)$ & $<0.001$ \\
\hline Glu(IQR) & $5.3(4.7,6.3)$ & $5.4(4.7,6.4)$ & 0.571 \\
\hline $\mathrm{Cr}[(\mathrm{umol} / \mathrm{L}), \mathrm{IQR}]$ & $73.2(62.7,85.8)$ & $71.2(60.5,83)$ & 0.459 \\
\hline EF\%, median(IQR) & $60(54,66)$ & $60(54.2,63.8)$ & 0.345 \\
\hline $\mathrm{RC}(\mathrm{mmol} / \mathrm{L}), \operatorname{median}(\mathrm{IQR})$ & $0.6(0.4,0.8)$ & $0.7(0.4,1.2)$ & $<0.001$ \\
\hline Ratio, median(IQR) & $2.7(2,4)$ & $3.3(1.9,5.4)$ & 0.124 \\
\hline
\end{tabular}

Abbreviations: COPD, chronic obstructive pulmonary disease; ACEl, angiotensin-converting enzyme inhibitors; N, neutrophils; GS scores, Gensini score; Hb, hemoglobin; PCT, platelet, procalcitonin; TC, total cholesterol; TG, triglycerides; HDL-C, high-density lipoprotein cholesterol; LDL-C, lowdensity lipoprotein cholesterol; RC, remnant cholesterol; RDW, the width of red blood cell volume distribution; ALT, alanine transaminase; Cr, creatinine; Ef\%, left ventricular systolic function.

Of CAD patients included in our study, 94 subjects (19.3\%) had ISR. Most of the baseline characteristics between the two groups were similar, such as gender, underlying disease (hypertension, diabetes, stroke), medication history (diuretic, ACEI), smoking, lesion location, ALT, Hb, PDW, RDW, MPV, PLT, TG, TC, TG, HDL-C, monocyte, neutrophil/ lymphocyte ratio and Cr. However, as Tables 1 shown, underlying disease (COPD), GS score, LDL-C, and RC showed significant differences between the groups (all $\mathrm{P}<0.05$ ).

The feature selection of the risk prediction model for ISR in patients after PCI is based on the 477 patients in the cohort (parameter selection is shown in Figure 2). 35 features were reduced to 5 potential predictors, and non-zero coefficients were used in the LASSO regression model. Those factors include: history of COPD, GS score of vascular assessments before PCI, monocyte, RC, and neutrophil/lymphocyte ratio. The Norman diagram based on the regression coefficient is shown in Figure 3.

33 33

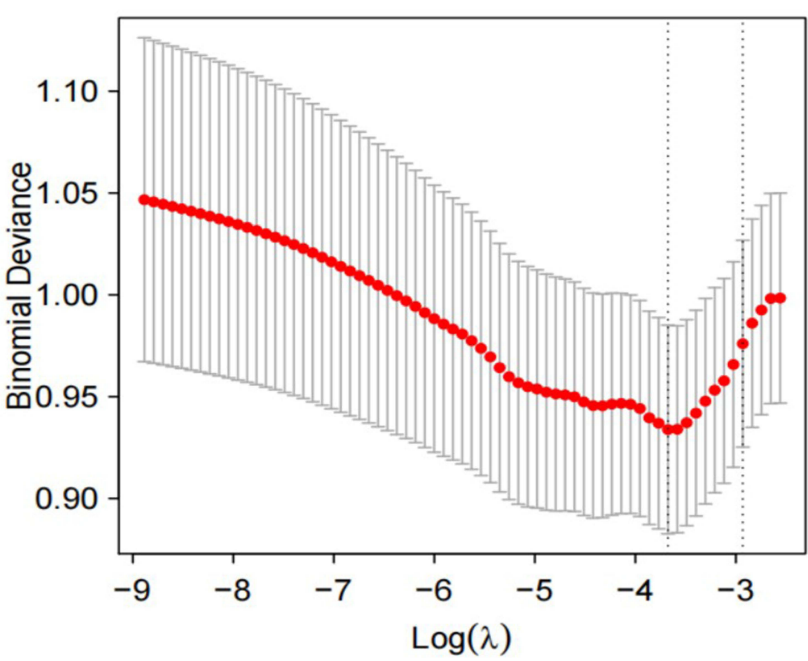

33

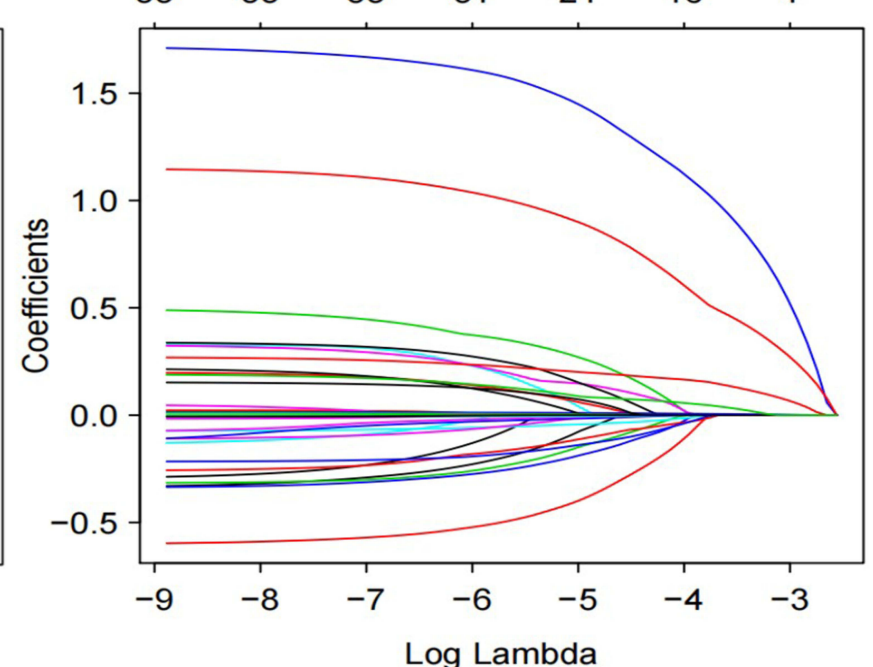

Figure 2 Risk factors selecting using LASSO model.

Notes: (Left panel) Optimal parameter (lambda) selection for the LASSO model was cross validated five the minimum criterion. Partial likelihood deviation (binomial deviation) curves versus log(lambda). Dotted vertical lines are drawn at the best values of ISE (I-SE criterion) using the minimum criterion and the maximum criterion. (Right) LASSO coefficient profiles for 35 characteristics. The coefficient profiles were produced from log (lambda) sequences.The vertical lines are drawn on the value selected using five fold cross validation, where the best lambda resulted in non-zero coefficients for five features. 
Points

$\begin{array}{rrrrrrrrrrr}0 & 10 & 20 & 30 & 40 & 50 & 60 & 70 & 80 & 90 & 100\end{array}$

COPD

1

COPD

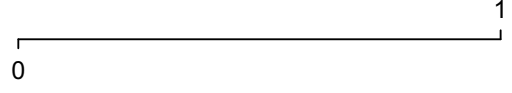

GS score

$\begin{array}{llllllllllll}0 & 20 & 40 & 60 & 80 & 100 & 120 & 140 & 160 & 180 & 200 & 220\end{array}$

Monocyte

\begin{tabular}{llllllllllll}
\hline 0 & 1 & 2 & 3 & 4 & 5 & 6 & 7 & 8 & 9 & 10 & 11
\end{tabular}

$\mathrm{RC}$

\begin{tabular}{llllllll}
\hline 0 & 0.5 & 1 & 1.5 & 2 & 2.5 & 3 & 3.5
\end{tabular}

ratio

\begin{tabular}{llllllllll}
\hline 0 & 2 & 4 & 6 & 8 & 10 & 12 & 14 & 16 & 18
\end{tabular}

Total Points

$\begin{array}{lllllllllllll}0 & 20 & 40 & 60 & 80 & 100 & 120 & 140 & 160 & 180 & 200 & 220 & 240\end{array}$

Risk of result

\begin{tabular}{llllll}
\hline 0.05 & 0.2 & 0.4 & 0.6 & 0.8 & 0.95
\end{tabular}

Figure 3 Nomogram to predict the probability of ISR in patients with stent implantation.

Notes: The nomogram included a medical history of COPD, GS score of vascular assessment before PCI, RC, monocyte, neutrophil/lymphocyte ratio.

Abbreviations: COPD, chronic obstructive pulmonary disease; GS scores, Gensini score; RC, remnant cholesterol.

The binary multivariate logistic regression comprised features selected from the lasso-penalized regression analysis (Table 2). The 5 independent risk factors for ISR were COPD, GS score, monocyte, RC, and neutrophil/lymphocyte ratio. The collinearity diagnostic test indicated that there was no significant collinearity between the independent variables in the regression model, and the variance inflation factors (VIFs) were 1.012, 1.006, 1.024, 1.037, and 1.008, respectively (all VIFs $<10$ ). 
Table 2 Multivariable Logistic Regression Analysis of Predictors of ISR

\begin{tabular}{|l|c|c|c|}
\hline Characteristics & OR & $\mathbf{9 5 \%} \mathbf{~ C l}$ & P value \\
\hline COPD & 4.56 & $1.98-10.40$ & $<0.001$ \\
GS scores & 1.01 & $1.00-1.02$ & 0.002 \\
RC & 2.09 & $1.40-3.11$ & $<0.001$ \\
Monocyte & 1.3 & $1.04-1.70$ & 0.035 \\
Neutrophil/lymphocyte ratio & 1.11 & $1.01-1.21$ & 0.021 \\
\hline
\end{tabular}

Abbreviations: $\mathrm{Cl}$, Confidence interval; COPD, chronic obstructive pulmonary disease; RC, remnant cholesterol.

Internal validation was performed with 1000 repeats, and the results were consistent. In addition, the AUC of the prediction model was 0.841 (Figure 4). A Hosmer-Lemeshow goodness-of-fit test was performed to evaluate this prediction model, yielding $\mathrm{P}=0.609$, and a calibration curve was also provided in Figure 5, confirming no divergence between anticipated and observed probability. Figure 6 shows the decision curve analysis for the ISR nomogram. The results showed that the nomogram might be used to forecast the likelihood of ISR in patients having PCI with high accuracy and a broader range of threshold probabilities, and it could have clinical implications.

\section{Discussion}

PCI is the mainstay of revascularization in patients with coronary artery disease, and in-stent restenosis remains a problem that greatly affects the long-term prognosis of post-PCI patients. The safety of stent implantation has significantly increased in recent years as a result of technological developments. ISR, on the other hand, remains one of the most significant issues. The ISR rate reached 19.7\% (94/477) in our research, which was consistent with previous studies (3-20\%). ${ }^{3}$ Life-threatening consequences can arise should the ISR continue to deteriorate without prompt recognition and treatment. Therefore, early identification of risk factors for ISR is crucial in preventing major

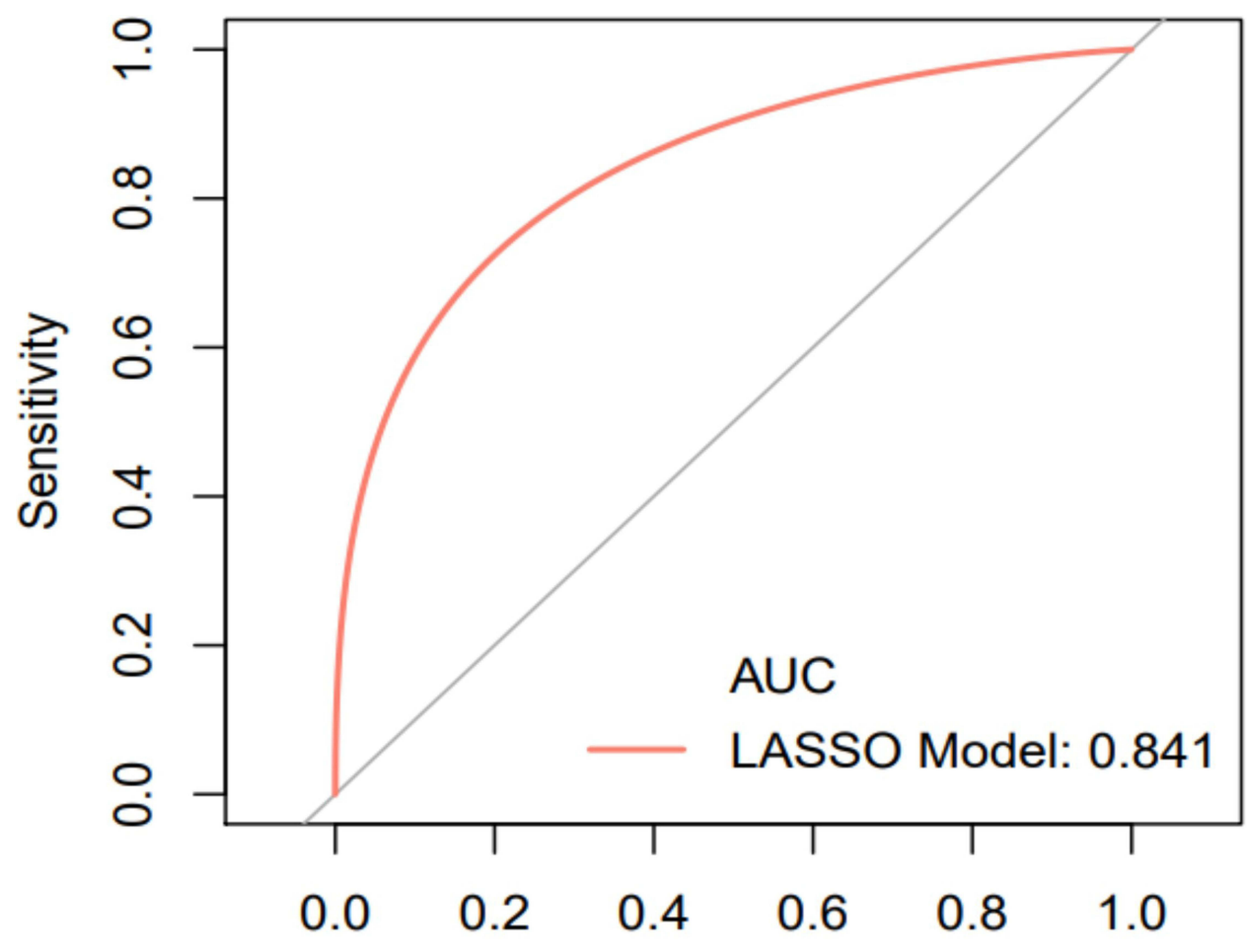

Figure 4 ROC curves for validating the discrimination power of nomogram. Abbreviation: ROC, receiver operator characteristic. 


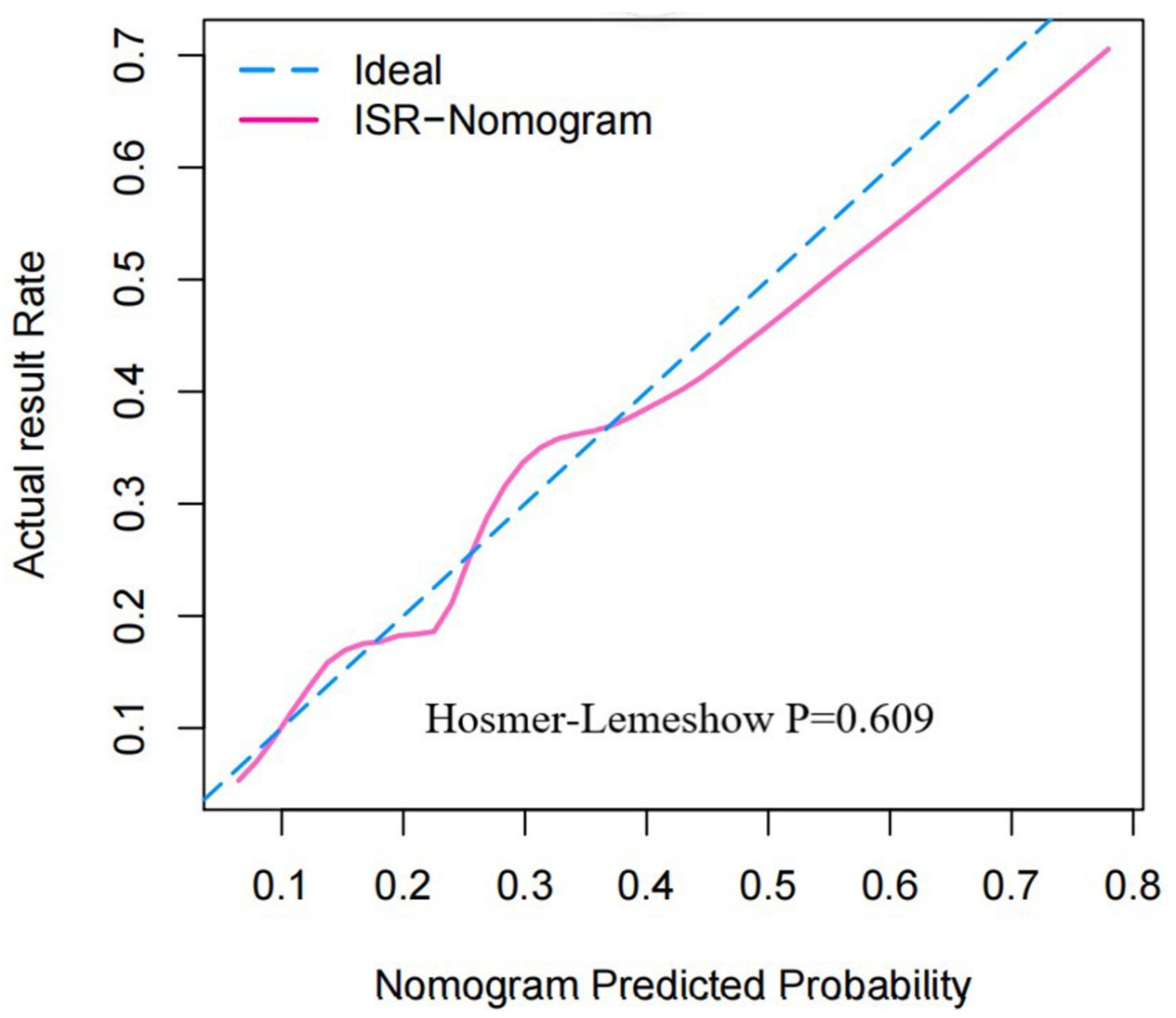

Figure 5 Calibration plots of the nomogram for the probability of PCl patients with ISR.

Notes: The x-axis represents prediction probability. The $y$-axis represents the actual probability. The diagonal dotted line represents a perfect prediction by an ideal model. The solid line represents the performance of the nomogram, of which a closer fit to the diagonal dotted line represents a better prediction.

postoperative complications. Herein, we firstly developed a nomogram utilizing the five preoperative predictors from the multivariate analysis: a medical history of COPD, monocytes, GS score, RC, and neutrophil/lymphocyte ratio.

Previous studies $^{5,6}$ summarized the predictors of ISR as follows: medication history (eg, clopidogrel), prior PCI, stent characteristics, and some indicators concerning inflammatory responses and lipid metabolism (eg, TC, LDL-C, CRP, monocyte). ${ }^{11}$

Similar to the previous studies, ${ }^{11}$ we also found that monocytes can be one of the independent risk factors for ISR. Nan et al reported that activated monocytes release large amounts of pro-inflammatory cytokines, and then cause vasoconstriction and non-specific recruitment, proliferation, and activation of other cells, including vascular smooth muscle cells in the vascular wall, which may account for the results in our study.

Another systemic factor noted in our study to be closely related to ISR after PCI is preoperative neutrophil/ lymphocyte ratio, the index associated with inflammatory response and neointimal proliferation, ${ }^{4,5,17,18}$ which was consistent with the previous research that neutrophil/lymphocyte is a strong inflammatory marker ${ }^{19-22}$ and closely associated with Cardiovascular disease. In contrast to previous reports, we also discovered that RC had a higher predictive value for ISR than LDL-C. According to relevant literature, ${ }^{7,23,24}$ an increase in fasting RC level increases the degree of coronary atherosclerosis stenosis and RC may be a better indicator of lipid metabolism in the body than LDL cholesterol.

Interestingly, we also found that patients with chronic obstructive pulmonary disease may have a higher risk of developing ISR. According to relevant literature, ${ }^{9,25-28}$ this could be due to the fact that COPD and CVD have similar risk factors. Low-grade systemic inflammation is one of the primary processes that may be responsible for the systemic impacts on distant illnesses and the increased rate of comorbidity, particularly cardiovascular comorbidity, in COPD 


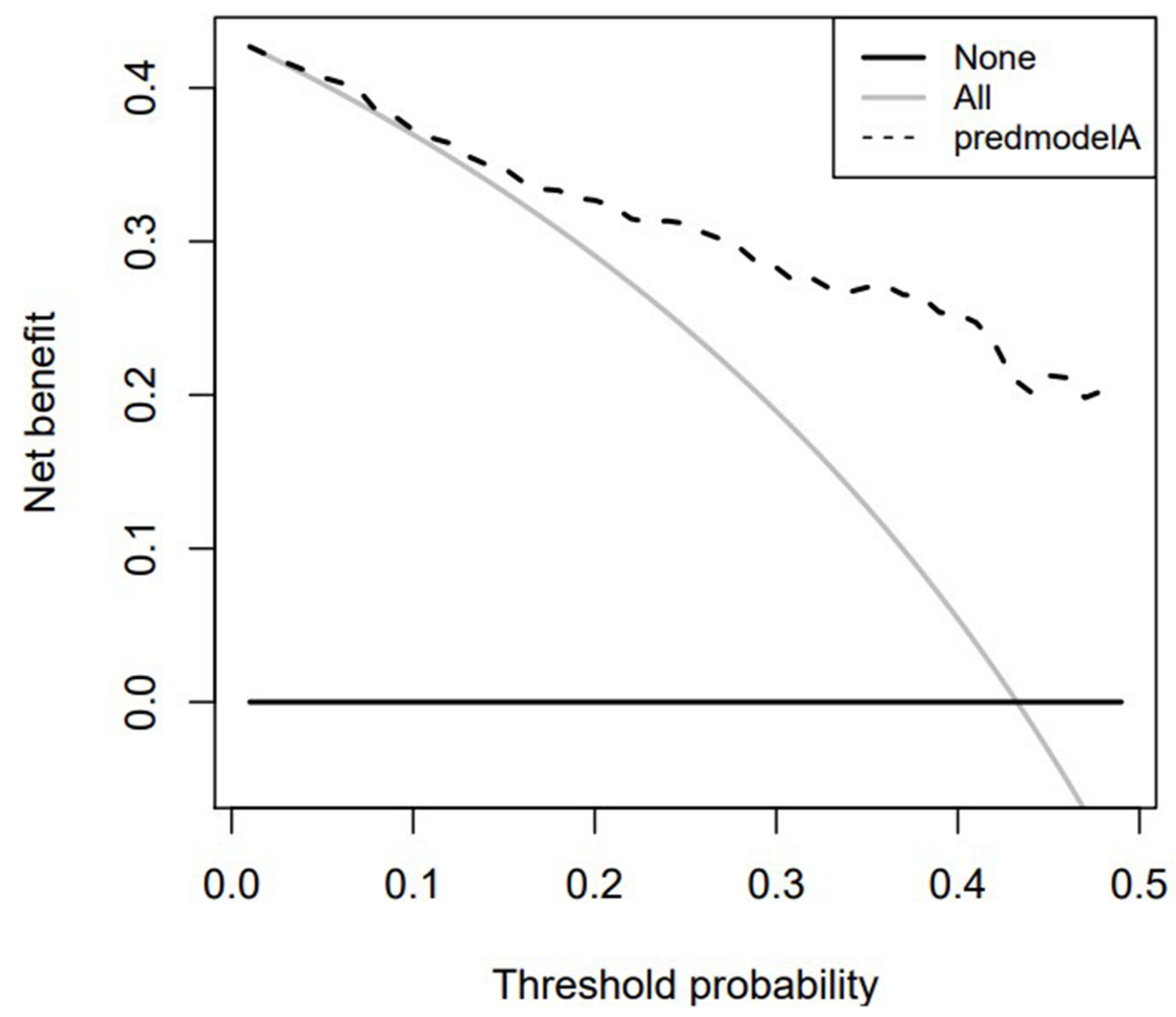

Figure 6 Decision curve analysis for the ISR prediction nomogram.

Notes: The ISR risk nomogram is represented by the dotted line. The thin solid line denotes the expectation that all patients are affected by ISR. The thick solid line denotes the assumption that there are no patients with ISR. When the threshold probability is $>4.5$, decision curve analysis demonstrates that adopting this ISR prediction nomogram has a net benefit.

patients. ${ }^{28}$ Regretfully, no relevant mechanistic investigations have been conducted to explain why a history of COPD can predict the occurrence of ISR, which will be the subject of our next research.

The most interesting finding was that GS score is an independent predictor of ISR. It is known that the morphological characteristics of the diseased vessel, the location of the lesion, and the degree of stenosis can make PCI procedures more difficult and increase the risk of preoperative vascular injury, leading to ISR. These factors have been shown to be independent risk factors for ISR, ${ }^{2}$ but no quantitative assessments of diseased vessels have been considered in any published models and no studies have examined whether the GS score, which combines these characteristics, is a strong predictor of ISR. And we improved that GS score is a strong predictor of ISR in CAD patients for the first time.

And in previous studies, ${ }^{8,29,30}$ the level of the prognostic utility of the ISR prediction model was still not entirely satisfactory, with a C-statistic below 0.7. We, therefore, developed a predictive model based on the GS score, a proxy for coronary lesion factors, ${ }^{31,32}$ and other predictors for patients undergoing PCI in the Enshi region.

Nevertheless, the research still has several limitations. First of all, its validity is limited by the small sample size and the low number of events although our sample size had met the required the minimum sample size of building the model 0.80 is 140. Furthermore, this was a single-Center study with no external validation. Despite an internal validation, the prediction model's generalization may be compromised. Lastly, the study had a retrospective design with an inadequate level of evidence.

\section{Conclusion}

Above all, in the Enshi population, we created a new prediction model based on the history of COPD, GS score of vascular assessment before PCI, monocyte, RC, and neutrophil/lymphocyte ratio, to help clinicians discern high-risk ISR patients, optimize treatment strategy, thus improve the prognosis of these patients. Furthermore, we visualized the 
prognostic risk factors in LASSO regression by using nomogram, evaluated the accuracy and clinical practicability of the predictive model by using DCA and Calibration curve. And it is found that the model is satisfactory in terms of goodness of fit, clinical usefulness, and accuracy.

\section{Abbreviations}

ISR, in-stent restenosis; DES, drug-eluting stents; PCI, percutaneous coronary intervention; LASSO, least absolute shrinkage and selection operator; ROC, receiver operator characteristic; RC, residual cholesterol; GS score, Gensini score; TC, total cholesterol; DCA, decision curve analysis; CHD, coronary heart disease; COPD, chronic obstructive pulmonary disease; PDW, platelet distribution width; MPV, mean platelet volume; GLU, glucose; N, neutrophils; LY, lymphocyte; MNC, monocyte; Hb, hemoglobin; PLT, platelet count; TC, total cholesterol; TG:triglycerides; HDL-C: high-density lipoprotein cholesterol,; LDL-C, low-density lipoprotein cholesterol; RDW, width of red blood cell volume distribution; ALT, alanine transaminase; AST, aspartate transaminase; LpA1, lipoprotein A1; LpB1, lipoprotein B1; Apo a, apolipoprotein a; $\mathrm{Cr}$, creatinine; $\mathrm{TSH}$, thyroid-stimulating hormone; $\mathrm{EF} \%$, left ventricular ejection fraction; $\mathrm{SD}$, standard deviation; PCT, procalcitonin.

\section{Data Sharing Statement}

All relevant data supporting the conclusions of this article are included within the article.

\section{Ethical Approval}

The authors are accountable for all aspects of the work in ensuring that questions related to the accuracy or integrity of any part of the work are appropriately investigated and resolved. Written informed consent was obtained from the patient for publication of this study and any accompanying images. The study was approved by Ethical Committees of Central Hospital of Enshi Tujia and Miao Autonomous Prefecture. The data are anonymous, and the requirement for informed consent was therefore waived.

\section{Author Contributions}

All authors made substantial contributions to conception and design, acquisition of data, or analysis and interpretation of data; took part in drafting the article or revising it critically for important intellectual content; agreed to submit to the current journal; gave final approval of the version to be published; and agree to be accountable for all aspects of the work.

\section{Funding}

This work was supported in part by the National Natural Science Foundation of China (82160072) and the Science and Technology Support Project of Enshi Science and Technology Bureau (D20210024). The funding bodies played no role in design of the study, collection, analysis, and interpretation of the data and in writing the manuscript.

\section{Disclosure}

Yinhua Luo and Ni Tan are co-first authors for this study. The authors report no conflicts of interest in this work.

\section{References}

1. Khattab MH, Sherry AD, Barker CM. The birth, decline, and contemporary re-emergence of endovascular brachytherapy for prevention of in-stent restenosis. Brachytherapy. 2021;20(2):485-493. doi:10.1016/j.brachy.2020.09.012

2. Singh AD, Singal AK, Mian A, et al. Recurrent Drug-Eluting Stent In-Stent Restenosis: a State-of-the-Art Review of Pathophysiology, Diagnosis, and Management. Cardiovasc Revasc Med. 2020;21(9):1157-1163. doi:10.1016/j.carrev.2020.01.005

3. Lee MS, Banka G. In-stent Restenosis. Interv Cardiol Clin. 2016;5(2):211-220. doi:10.1016/j.iccl.2015.12.006

4. Bakogiannis C, Sachse M, Stamatelopoulos K, et al. Platelet-derived chemokines in inflammation and atherosclerosis. Cytokine. 2019;122:154157. doi:10.1016/j.cyto.2017.09.013

5. Gai M-T, Zhu B, Chen X-C, et al. A prediction model based on platelet parameters, lipid levels, and angiographic characteristics to predict in-stent restenosis in coronary artery disease patients implanted with drug-eluting stents. Lipids Health Dis. 2021;20(1):118. doi:10.1186/s12944-021-01553-2 
6. He W, Xu C, Wang X, et al. Development and validation of a risk prediction nomogram for in-stent restenosis in patients undergoing percutaneous coronary intervention. BMC Cardiovasc Disord. 2021;21(1):435. doi:10.1186/s12872-021-02255-4

7. Lorenzatti AJ, Toth PP. New Perspectives on Atherogenic Dyslipidaemia and Cardiovascular Disease. Eur Cardiol. 2020;15:1-9. doi:10.15420/ ecr.2019.06

8. Stolker JM, Kennedy KF, Lindsey JB, et al. Predicting restenosis of drug-eluting stents placed in real-world clinical practice: derivation and validation of a risk model from the EVENT registry. Circ Cardiovasc Interv. 2010;3(4):327-334. doi:10.1161/CIRCINTERVENTIONS.110.946939

9. Almagro P, Boixeda R, Diez-Manglano J, et al. <p>Insights into Chronic Obstructive Pulmonary Disease as Critical Risk Factor for Cardiovascular Disease. Int J Chron Obstruct Pulmon Dis. 2020;15:755-764. doi:10.2147/COPD.S238214

10. Liu Y, Imanishi T, Ikejima H, et al. Association between circulating monocyte subsets and in-stent restenosis after coronary stent implantation in patients with ST-elevation myocardial infarction. Circ J. 2010;74(12):2585-2591. doi:10.1253/circj.CJ-10-0544

11. Nan J, Meng S, Hu H, et al. The Predictive Value of Monocyte Count to High-Density Lipoprotein Cholesterol Ratio in Restenosis After Drug-Eluting Stent Implantation. Int J Gen Med. 2020;13:1255-1263. doi:10.2147/IJGM.S275202

12. Afari ME, Bhat T. Neutrophil to lymphocyte ratio (NLR) and cardiovascular diseases: an update. Expert Rev Cardiovasc Ther. $2016 ; 14(5): 573-577$. doi:10.1586/14779072.2016.1154788

13. Yang Y, Ge F, Shen J, et al. The relationship between neutrophil-lymphocyte ratio and in-stent restenosis in superficial femoral artery. Biosci Rep. 2020;40:7. doi:10.1042/BSR20193448

14. Castañer O, Pintó X, Subirana I, et al. Remnant Cholesterol, Not LDL Cholesterol, Is Associated With Incident Cardiovascular Disease. J Am Coll Cardiol. 2020;76(23):2712-2724. doi:10.1016/j.jacc.2020.10.008

15. Varbo A, Nordestgaard BG. Remnant lipoproteins. Curr Opin Lipidol. 2017;28(4):300-307. doi:10.1097/MOL.0000000000000429

16. Rampidis GP, Benetos G, Benz DC, et al. A guide for Gensini Score calculation. Atherosclerosis. 2019;287:181-183. doi:10.1016/j. atherosclerosis.2019.05.012

17. Hayıroğlu M, Çınar T, Çinier G, et al. Evaluating systemic immune-inflammation index in patients with implantable cardioverter defibrillator for heart failure with reduced ejection fraction. Pacing Clin Electrophysiol. 2022;45(2):188-195. doi:10.1111/pace.14436

18. Cakir Guney B, Hayiroglu M, Senocak D, et al. Evaluation of N/LP Ratio as a Predictor of Disease Progression and Mortality in COVID-19 Patients Admitted to the Intensive Care Unit. Medeni Med J. 2021;36(3):241-248. doi:10.5222/MMJ.2021.95676

19. Balta S, Celik T, Mikhailidis DP, et al. The Relation Between Atherosclerosis and the Neutrophil-Lymphocyte Ratio. Clin Appl Thromb Hemost. 2016;22(5):405-411. doi:10.1177/1076029615569568

20. Shen H, Dai Z, Wang M, et al. Preprocedural Neutrophil to Albumin Ratio Predicts In-Stent Restenosis Following Carotid Angioplasty and Stenting. J Stroke Cerebrovasc Dis. 2019;28(9):2442-2447. doi:10.1016/j.jstrokecerebrovasdis.2019.06.027

21. Wang Z, Liu C, Fang H. Blood Cell Parameters and Predicting Coronary In-Stent Restenosis. Angiology. 2019;70(8):711-718. doi:10.1177/ 0003319719830495

22. Zhu G, Li Z, Yuan L, et al. The Relationship Between Neutrophil-Lymphocyte Ratio and In-Stent Restenosis. Angiology. 2018;69(7):642-643. doi:10.1177/0003319718762913

23. Li B, Wang A, Wang Y, et al. A study on the correlation between remnant cholesterol and urinary albumin to creatinine ratio in Chinese community adults: a report from the REACTION study. J Diabetes. 2020;12(12):870-880. doi:10.1111/1753-0407.13076

24. Maranhão RC, Pala D, Freitas FR. Lipoprotein removal mechanisms and aging: implications for the cardiovascular health of the elderly. Curr Opin Endocrinol Diabetes Obes. 2020;27(2):104-109. doi:10.1097/MED.0000000000000529

25. Januszek R, Siudak Z, Dziewierz A, et al. Chronic obstructive pulmonary disease affects the angiographic presentation and outcomes of patients with coronary artery disease treated with percutaneous coronary interventions. Pol Arch Intern Med. 2018;128(1):24-34. doi:10.20452/pamw.4145

26. Farb A, Sangiorgi G, Carter AJ, et al. Pathology of acute and chronic coronary stenting in humans. Circulation. 1999;99(1):44-52. doi:10.1161/01. CIR.99.1.44

27. Onishi K. Total management of chronic obstructive pulmonary disease (COPD) as an independent risk factor for cardiovascular disease. J Cardiol. 2017;70(2):128-134. doi:10.1016/j.jjcc.2017.03.001

28. Sinden NJ, Stockley RA. Systemic inflammation and comorbidity in COPD: a result of 'overspill' of inflammatory mediators from the lungs? Review of the evidence. Thorax. 2010;65(10):930-936. doi:10.1136/thx.2009.130260

29. Stolker JM, Cohen DJ, Kennedy KF, et al. Combining clinical and angiographic variables for estimating risk of target lesion revascularization after drug eluting stent placement. Cardiovasc Revasc Med. 2017;18(3):169-176. doi:10.1016/j.carrev.2016.12.014

30. Yeh RW, Normand S-LT, Wolf RE, et al. Predicting the restenosis benefit of drug-eluting versus bare metal stents in percutaneous coronary intervention. Circulation. 2011;124(14):1557-1564. doi:10.1161/CIRCULATIONAHA.111.045229

31. Avci A, Fidan S, Tabakçı MM, et al. Association between the Gensini Score and Carotid Artery Stenosis. Korean Circ J. 2016;46(5):639-645. doi:10.4070/kcj.2016.46.5.639

32. Morice MC. Outcomes in patients with de novo left main disease treated with either percutaneous coronary intervention using paclitaxel-eluting stents or coronary artery bypass graft treatment in the Synergy Between Percutaneous Coronary Intervention with TAXUS and Cardiac Surgery (SYNTAX) trial. Circulation. 2010;121(24):2645-2653. doi:10.1161/CIRCULATIONAHA.109.899211

International Journal of General Medicine

Dovepress

\section{Publish your work in this journal}

The International Journal of General Medicine is an international, peer-reviewed open-access journal that focuses on general and internal medicine, pathogenesis, epidemiology, diagnosis, monitoring and treatment protocols. The journal is characterized by the rapid reporting of reviews, original research and clinical studies across all disease areas. The manuscript management system is completely online and includes a very quick and fair peer-review system, which is all easy to use. Visit http://www.dovepress.com/testimonials.php to read real quotes from published authors.

Submit your manuscript here: https://www.dovepress.com/international-journal-of-general-medicine-journal 